\title{
RUPTURA HEPATICA - Reporte de 11 Casos
}

\author{
Dr. Jaime Cantillo G.* \\ Dra. Laura Rojas R.**
}

\section{Introducción}

La ruptura hepática espontánea complicación poco frecuente pero gravemente mortal en Obstetricia es afortunadamente rara entre nosotros ya que solo 20 casos se han publicado en el transcurso de 15 años. Motivo de esta publicación son 11 casos más.

Después del bazo, es el hígado la víscera que más se rompe (1). Su número ha aumentado enormemente por causas traumáticas (accidentes de trabajo, tráfico, (2) heridas por armas corto-punzantes, o de fuego, en las guerras, etc.). En la Guerra del Vietnam, esto ha servido para perfeccionar técnicas quirúrgicas y materiales adecuados para cohibir la hemorragia con gran éxito $(3,4,5,6,7)$.

Pero no solamente son las causas traumáticas las que originan la ruptura hepática, pues esta puede estar asociada a enfermedades como paludismo, fiebre tifoidea, sífilis, enfermedades vasculares (8) y tumorales.

En la mujer la ruptura hepática puede ocurrir en el curso de la gestación normal 0 , especialmente, si ésta se complica con toxemia $(9,10)$.

Abercrombie en 1844, (Inglaterra) fue el primero que comunicó un caso en una mujer embarazada (11), Pal- tauf (1888), 40 años más tarde, publica el segundo caso (12), posteriormente varios autores europeos reportan algunos casos esporádicos, tanto que hasta 1930 se habían publicado 9 en total $(13,14)$.

\section{Material y Métodos}

Suministramos 11 casos más, extraídos de las historias clínicas y de los archivos de Patología del Instituto Materno Infantil de Bogotá, Colombia, desde el 1․ de Enero de 1966 al 31 de Diciembre de 1972

Durante este lapso se registraron 214.750 ingresos con 156.185 partos, 42.093 abortos, y se realizaron 1.123 autopsias.

De los 11 casos extraídos sde este material, 9 tienen estsudio de necropsia. Dos carecen de ella, pero en base a los datos de descripción quirúrgica, bastante claros, consideramos que debían incluirse en el presente estudio. En las Tablas 1 y 2 están consignados los datos más importantes de la historia clínica y autopsia de cada uno. La Foto № 1 muestra el aspec-

\footnotetext{
* Profesor Asistente del Departamento de Anatomía Patológica de la Facultad de Medicina de la Universidad Nacional.

** Profesor Asistente del Departamento de Ginecología y Obstetricia de la Facultad de Medicina de la Universidad Nacional.
} 


\begin{tabular}{|c|c|c|c|c|c|c|c|c|}
\hline \multicolumn{9}{|c|}{$R \cup P T U R A \quad H E P A T I C A$} \\
\hline CNO & $\begin{array}{c}N^{2} \\
\text { AUTOPSIA }\end{array}$ & EDAD & E. EMB. & SINTOMAS & SIGNOS & DX. DE INGRESO & EVOLUCION & TRATAMIENTO \\
\hline । & $190-66$ & 33 & $27-s$ & Epigostralgia & Shock & Toxemia Infarto & Fallece a la 2 horas & $\begin{array}{l}\text { Cesárea post-mortem. Ruptura hepá- } \\
\text { tica (hemoperitoneo } 2.000 \mathrm{ctms} \text { ). }\end{array}$ \\
\hline 2 & $299-66$ & 20 & $40-5$ & Epigaltralgia & T.A $190 \times 110$ & Eclampsia & $\begin{array}{l}\text { Continua Eclampsia y } \\
\text { fallece }\end{array}$ & Médico \\
\hline 3 & $88-67$ & 36 & $36-s$ & Cefáleo & $\begin{array}{l}\text { Edemas T.A. } \\
200 \times 100\end{array}$ & Preeclampsia & $\begin{array}{l}\text { Hemorragía por placento previa } \\
\text { Paro cardiaco en cirugía }\end{array}$ & Cesárea \\
\hline 4 & $15-69$ & 20 & $37-5$ & Epigastralgia & T.A. $160 \times 120$ & Eclampsia & Fallece a las 6 horas & Médico \\
\hline 5 & $59-69$ & 40 & $32-s$ & Epigastralgia & T.A. $160 \times 110$ & E.V.C.H. Toxemia & Parto espontáneo Shock I.R.A. & Hepatorrafia \\
\hline 6 & $71-69$ & 32 & $8-5$ & $\begin{array}{l}\text { Hemorragia } \\
\text { Genital }\end{array}$ & $\begin{array}{l}\text { Defensa } \\
\text { Abdominal }\end{array}$ & Abor to. Pelvi-peritonitis & Entra en Shock & $\begin{array}{l}\text { Liquemine. Salpingooforectomía } \\
\text { izquierdo por embarazo ectopico } \\
\text { roto }\end{array}$ \\
\hline 7 & $53-71$ & 36 & $38-s$ & Epigastralgia & $\begin{array}{l}\text { T.A. } 220 \times 120 \\
\text { Hepatomegalia }\end{array}$ & Inminencia de eclampsia & Shock hipovolémico & Hepatorrafía Cesárea \\
\hline 8 & $86-71$ & 38 & $40-s$ & Epigastralgia & $\begin{array}{l}\text { Shock Hepato- } \\
\text { megalia }\end{array}$ & Ruptura hepática & - & $\begin{array}{l}\text { Hepatorrafía. Cesáreo. Histe- } \\
\text { rectomía }\end{array}$ \\
\hline 9 & $196-72$ & 56 & - & Prolapso & Prolapso III & Prolapso grado III & - & Histerectomía Vaginal \\
\hline 10 & - & 35 & $36-5$ & Epigastralgia & Shock & Ruptura uterina o hepótica & Shock & $\begin{array}{l}\text { Hepatorratio. Cesárea. Hemoperitoned } \\
(4.000 \mathrm{cc}) \text {. }\end{array}$ \\
\hline 11 & - & 25 & $24-s$ & Epigastralgia & $\begin{array}{l}\text { Convulsiones } \\
\text { T.A. } 190 \times 130\end{array}$ & Eclampsia & Parto espontáneo. Shock & $\begin{array}{l}\text { Hepatorrafia. Hemoperitoneo } \\
(3.000 \mathrm{cc}) \text {. }\end{array}$ \\
\hline
\end{tabular}




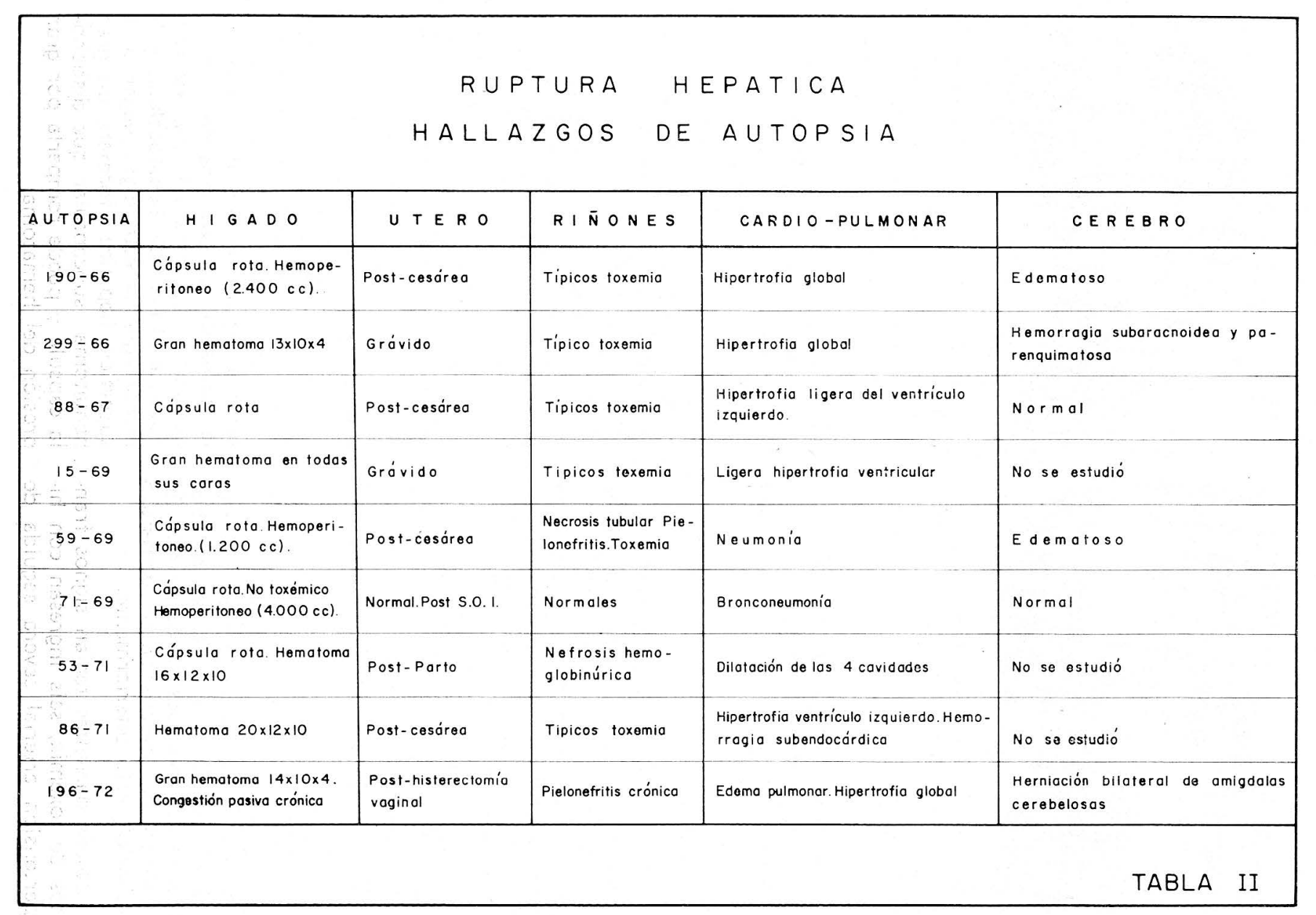




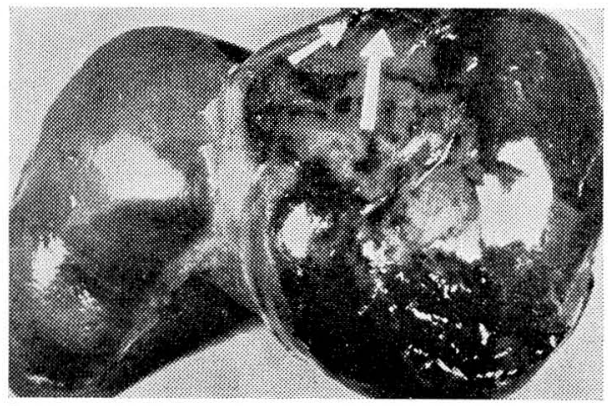

FOTO $N^{9} 1$ - Hematoma subcapsular de hígadc. Las flechas muestran ruptura de la cápsula, ocasionando un hemoperitoneo que causó la muerte de una de nuestras pacientes eclámpticas.

to macroscópico del hígado del Caso N. 1 que corresponde a la autopsia 190-66, y la 2 al cas oN: 4, autopsia 15-69.

\section{Resultados}

El accidente ocurrió en 10 pacientes que cursaban embarazo y uno en una paciente de 56 años que se intervino con histerectomía vaginal por prolapso genital III. La edad mínima fue de 20 años con una edad promedio de 29.5 años.

Todas las pacientes eran multigestantes excepto una G1 P0 con embarazo de más o menos 40 semanas quien padece de eclampsia y muere por A.C.V.H. y ruptura de hígado, hallazgo de autopsia.

Nueve pacientes se hallaban en el último trimestre de su gestación. Una cursaba apenas la 8a semana de embarazo durante el cual se practica maniobras abortivas (Caso № 8). En realidad se trataba de un embarazo tubárico, roto ulteriormente.

Nueve pacientes tenían signos francos de toxemia, seis ingresan con hipertensión arterial severa seguida de

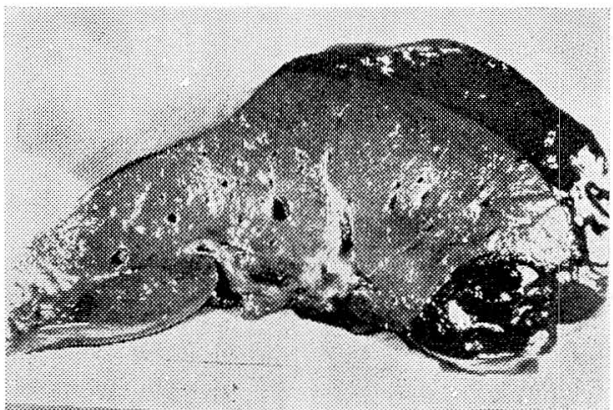

FOTO $\mathbf{N}^{0} 2$ - Superficie de corte de hígado. Obsérvese el gran hematoma que cubre ambas caras del lóbulo derecho y parte de la cara suparior del lóbulo izquierdo.

shock en el momento de la ruptura, tres ingresan en el estado de shock hipovolémico lo que facilitó el diagnóstico. Dos sufren el accidente después del parto espontáneo.

Seis pacientes fueron tratadas con laparotomía y hepatorrafia seguido de cesárea. Todas fallecen.

A la autopsia se comprobó toxemia en 7 de las pacientes embarazadas. Seis pacientes tenían gran hematoma subcapsular y en cinco se encontró éste asociado a hemoperitoneo que osciló entre 1.200 y 4.000 cc., de sangre. En todos los anteriores, la cápsula de Glisson se encontró rota.

\section{Discusión}

El mecanismo por el cual se rompe el hígado en las embarazadas con toxemia se explica por el vasoespasmo arteriolocapilar, la labilidad vascular, la formación de trombos de fibrina que con la isquemia y la hipoxia conducen a la necrosis hepatocelular y la hemorragia, si ésta logra llegar a la superficie hepática formará un gran hematoma subcapsular que distiende la cápsula y puede romperla por gran presión del hematoma. 
Según McKay y otros autores (15, 16, 17), el mecanismo por el cual se forma el hematoma se iniciaría a partir de los múltiples trombos de fibrina formados súbitamente en las arteriolas y sinusoides hepáticos; debido a la circulación de suplencia del hígado estos sinusoides y arteriolas comienzan a distenderse y a romperse por la labilidad vascular ocasionada por la misma toxemia conduciendo también a la necrosis hepatocelular y la hemorragia. A esto se le agregarían factores como la contracción del diafragma y de los músculos de la pared abdominal, la contracción uterina y el aumento de la presión intraabdominal; un hígado severamente lesionado por la necrosis hemorrágica subcapsular e intraparenquimatosa sería el terreno abonado para producir hematomas y rupturas espontáneas en las toxémicas; estos hechos nos explicarían nuestros casos de toxemia pero no explicaría los casos 8 y 11 cuyos hígados se rompieron espontáneamente según los datos de las historias clínicas. El uso de heparina en el caso No 8 no explica la producción del hematoma.

En cuanto al caso № 11 es posible que la hipertensión, debida a una nefroesclerosis, sumada a hipoxia durante 3 intervenciones consecutivas, explique la producción de él.

El tratamiento de la ruptura hepática sería siempre el quirúrgico con aplicación de sangre, drenaje del hematoma, hepatorrafia y colocación de substancias absorbentes, adhesivas y hemostáticas, para impedir la hemorragia de la superficie cruenta del hígado ("N-butyl Cyano Crylate adhesive Spray, Gelfoam, Oxycel").

En América, la primera publicación la hace Roblee (18), en 1940, después ha venido aumentando la casuística tanto en EE. UU. como en Lati- noamérica. Según la revisión de $H$. Villegas y col. en 1970 (19), la totaliclad de casos publicados en el mundo era de 75 asociados con embarazo y toxemia y la autora agregó 6 casos más. Sumando a los nuestros, los casos de Gómez P. y Peralta C. serían 97 en total.

En nuestro país, Colombia, hasta la fecha solo se han reportado 9 casos; la primera publicación la hizo Sánchez Torres (20), en 1963, en una paciente que falleció previo el diagnóstico positivo. Peralta Cayón (21) relata 3 muertes por esta causa. Riaño, una (22), Gómez Palacino (23), nos suministra la mayor casuística de 4 pacientes que sobrevivieron después del embarazo y la intervención quirúrgica.

\section{Resumen}

Se presentan 11 casos de ruptura hepática recolectados en el lapso que va comprendido de 1966 hasta 1972, algunos con hematoma subcapsular y hemoperitoneo, se hace un esbozo de la fisiopatología de la ruptura. Se realiza la revisión total de la literatura colombiana y parcial de la literatura universal.

\section{BIBLIOGRAFIA}

1 TIEDEMANN, R. N., MESHERRY, CH. K., and GLENN, F.: Lever hematoma after Blunt Injury. Am. J. Surgery. 124: 94, 1972.

2 LUCAS, C. E., and WALT, A. J.: Critical decisions in liver trauma, experience based on 604 cases. Arch. Surg. 101: 277, 1970.

3 WILSON, D. H.: Incidence aetiology, diagnosis and prognosis of closed abdominal injuries: a study of 265 consecutive cases. Brit. J. Surg. 50: 381, 1963.

4 ALBO, D., Jr. et al: Masive liver rtauma involving the suprarenal vena cava. Am. J. Surgery. 118: 960, 1969.

5 SCHOROCK, T., BLAIDELL, W. F. and MATHEWSON, C., Jr.: Management of blunt. 
Trauma to the liver and hepatic veins. Arch. Surg. 96: 698, 1968.

6 GOLDSMITH, N. A., and WOOD BURNE, R. T.: The surgical Anatomy Pertaining to liver resection. Surgery Gynec. Obstet. 105: 310. 1957.

7 HEISTER KAMP, CH. A. et al: Solid organ Injuries in Vietnam. Emergency hematosis with N-Butil cyanocrilate adhesive. Arch. Surg. 100: 109. 1970.

8 KATZENSTEIN, R. and RYAN, A. I.: Rupture of liver fatal hemorrage due to intrahepatic vascular disease. Report of two cases. J.A.M.A. 161: 199. 1956.

9 PORTNUFF, J. and BALLON. S.: Hepatic rupture in pregnancy. Am. J. Obst. Gynec. 114: 1102.1972.

10 BURTON BROWN, J., and SHEPHARD, J.: Rupture of the liver in eclampsia with fatal hemoperitoneum. Am. J. Obst. Gynec. 52: $325,1946$.

11 ABERCROMBIE, J.: Hemorrage of the liver. London Med. Gasette. 34: 792, 1844. Quated by Burton-Brown and Shepard.

12 PALTAUF, 1888 (Quated by Mahon).

13 MAHON, R.: La rupture spontanee du foie au cours de la grossesse. Rev. Franc. Gynec. et Obst. 58: 751, 1963.

14 DICKMANN, W. J.: The toxemia of pregnancy. Mosby St. Louis, 1952.

15 MCKAY, D. A.: Hematologic evidence of diseminated intravascular coagulation in eclampsia. Obstetrical and Gynecological

Survey. 27: 399. 1972.

16 WARDLE, E. N., and WRIGHT, N. A.: Role of fibrin in a model of pregnancy toxemia in the Rabbit. J. Obstet. Gynec. 115: 17, 1973 .

17 ANASTASIA, L. F., WILLIAMS, L. F., and BYRNE, J. J.: Cne bour hepatic ischemia without serious damage. J.A.M.A. 201 : 1051. 1967.

is ROBLEE, M. A.: Cited by Dieckmann N. J. Toxemia of pregnancy. St. Louis, the C. V. Mosby Company. 1941.

19 VILLEGAS, H. y col.: Ruptura hepática espontánea en la toxemia gravídica. Reporte cle seis casos y revisión de la literatura mundial. Ginec. Obst. Mex. 27: 543, 1970.

20 SANCHEZ TORRES, F.: Ruptura atraumática del hígado durante el embarazo. Una complicación más de la toxemia gravídica. Rev. Col. Obst. Ginec. 14: 367, 1963.

21 PERALTA CAYON, R.: Mortalidad Materna. Tribuna Médica. Bogotá. Colombia. V. 6 : (1) Mayo 15, 1967.

22 RIAÑO, M.:R uptura espontánea del hígado después del parto a término en embarazo asociado a toxemia. Rev. Col. Obst. Ginec. 20: 25, 1969.

23 GOMEZ PALACINO, J. A., LOZANO, C. A. Y MUÑOZ, L. A.: Ruptura hepática espontánea en gestantes con hipertensión arterial. Rev. Col. Obst. Ginec. 21: 23, 1970. 